\title{
Monodromy of projections of hypersurfaces
}

\author{
Maria Gioia Cifani ${ }^{1} \cdot$ Alice Cuzzucoli $^{2} \cdot$ Riccardo Moschetti $^{1}$
}

Received: 18 April 2020 / Accepted: 7 June 2021 / Published online: 17 June 2021

(C) The Author(s) 2021

\begin{abstract}
Let $X$ be an irreducible, reduced complex projective hypersurface of degree $d$. A point $P$ not contained in $X$ is called uniform if the monodromy group of the projection of $X$ from $P$ is isomorphic to the symmetric group $S_{d}$. We prove that the locus of non-uniform points is finite when $X$ is smooth or a general projection of a smooth variety. In general, it is contained in a finite union of linear spaces of codimension at least 2, except possibly for a special class of hypersurfaces with singular locus linear in codimension 1. Moreover, we generalise a result of Fukasawa and Takahashi on the finiteness of Galois points.
\end{abstract}

Keywords Monodromy $\cdot$ Projections $\cdot$ Uniform points $\cdot$ Focal points $\cdot$ Filling families

Mathematics Subject Classification $14 \mathrm{H} 30 \cdot 14 \mathrm{H} 50 \cdot 14 \mathrm{~J} 10 \cdot 14 \mathrm{~J} 70$

\section{Introduction}

This paper aims at studying the monodromy group of projections of irreducible and reduced complex projective hypersurfaces. Several accounts of this study, in particular in the case of projective curves, can be found in the literature. The classical Uniform Position Principle due to Castelnuovo, in the formulation of Harris [17], can be applied to show that the monodromy group of a general projection of a curve is the symmetric group. It has been proved in [13-15] that the monodromy group of an indecomposable projection of a general curve of genus greater than 3 is either the symmetric or the alternating group. More recently, all the monodromy groups of projections of a smooth planar curve of degree smaller or equal than 5 have been classified (see [23, 24, 29]).

This paper is motivated by the work of Pirola and Schlesinger [25], in which the authors consider the monodromy group of projections of any irreducible reduced

Riccardo Moschetti

riccardo.moschetti@unipv.it

Maria Gioia Cifani

mariagioia.cifani01@universitadipavia.it

Alice Cuzzucoli

a.cuzzucoli@warwick.ac.uk

1 Department of Mathematics 'F. Casorati', University of Pavia, via Ferrata 5, 27100 Pavia, Italy

2 Department of Mathematics, University of Warwick, Coventry, Warwickshire CV4 7AL, England 
projective curve. In our case, we fix a hypersurface $X \subset \mathbb{P}^{n+1}$ of degree $d$ and we consider all its natural linear projections $\pi_{P}$ from a point $P \notin X$. We classify such source points by means of the monodromy group of the associated projection. Our final goal is a characterisation of the locus of points for which the associated monodromy group is strictly contained in the symmetric group $S_{d}$.

Definition 1.1 The point $P$ is called uniform if $M\left(\pi_{P}\right) \cong S_{d}$ and non-uniform otherwise. We denote by $\mathcal{W}(X)$ the locus of non-uniform points of $X$.

Specifically, we are interested in two classes of non-uniform points: a point $P \in \mathcal{W}(X)$ is called Galois if the field extension associated with the map $\pi_{P}$ is a Galois extension. A point $P \in \mathcal{W}(X)$ is called decomposable if the projection $\pi_{P}$ is decomposable, i.e. if it factors via two morphisms of degree greater than 1 . The loci of Galois and decomposable points are denoted by $\mathcal{G}(X)$ and $\mathcal{D}(X)$, respectively.

The non-uniform locus $\mathcal{W}(X)$ is constructible and a first step in order to understand its structure is to compute its dimension. The uniform position principle extends to hypersurfaces by taking general hyperplane sections: indeed, for any irreducible, reduced hypersurface $X \subset \mathbb{P}^{n+1}$ we have $\operatorname{dim} \mathcal{W}(X)<n+1$. This bound is far from optimal. The case of curves has been solved by Pirola and Schlesinger: in the work [25], the authors proved that the locus of non-uniform points associated with projections of an irreducible reduced plane curve is finite. The case of surfaces in $\mathbb{P}^{3}$ has been partially covered in [7], where it is proved that the locus of non-uniform points of a smooth surface in $\mathbb{P}^{3}$ is finite. All these bounds are optimal.

The main result of this paper describes a property of the locus of non-uniform points for projective hypersurfaces:

Theorem 1.2 Let $X$ be an irreducible, reduced hypersurface of $\mathbb{P}^{n+1}, n \geq 2$. Then, $\mathcal{W}(X)$ is contained in a finite union of linear spaces of codimension 2 in $\mathbb{P}^{n+1}$, unless $X^{\text {sing }}$ is the union of at least 2 components isomorphic to $\mathbb{P}^{n-1}$. In this case, $\mathcal{W}(X)$ must be a union of rational curves, lying in the intersection of the tangent cones to points in $X^{\text {sing }}$.

Cones over planar curves which admit non-uniform points give examples of hypersurfaces $X$ for which $\mathcal{W}(X)$ has codimension 2 (see Example 4.6). The possibility of $\mathcal{W}(X)$ being a union of rational curves seems unlikely, and it is discussed in Remark 4.11. As a consequence of the main result, we give in Proposition 4.8 a bound on the dimension of the locus of non-uniform points for smooth varieties; in particular, such locus is finite for smooth hypersurfaces.

Theorem 1.3 Let X be a smooth, complex projective hypersurface of dimension $n$ in $\mathbb{P}^{n+1}$. Then, the locus of non-uniform points is finite.

The primary tool in the proofs is the theory of focal loci of families of lines in $\mathbb{P}^{n+1}$, a classical topic dating back to Segre ([26]). For our specific problem, we prove a generalisation of [6, Proposition 4.3] and of [8, Lemma 2], described in Lemma 3.7 and Lemma 3.8, respectively.

We also use the focal machinery to give a property of the monodromy group when $\mathcal{W}(X)$ is infinite and $X$ is not a cone. 
Theorem 1.4 If $\operatorname{dim} \mathcal{W}(X)>0$ and $X$ is not a cone, then the monodromy group associated with all but finitely many points of $\mathcal{W}(X)$ contains transpositions.

As a consequence of this result, we can give a characterisation of the two loci $\mathcal{G}(X)$ and $\mathcal{D}(X)$. Galois points have been introduced and extensively studied in various works, for instance $[10-12,30]$ to name a few. These works were particularly focused on computing the number of Galois points. Yoshihara gives many examples of smooth hypersurfaces $X$ with non-empty $\mathcal{G}(X)$ in [30, Proposition 11] (see also [12] for more general examples in case of normal hypersurfaces). We give a generalisation of a result of Fukasawa and Takahashi ([12, Proposition 6]).

Proposition 1.5 Let $X$ be an irreducible, reduced hypersurface in $\mathbb{P}^{n+1}$ of degree $d \geq 3$. Then $\mathcal{G}(X)$ is finite unless $X$ is a cone.

Decomposable maps were studied in many different contexts as in $[4,5]$. A consequence of Theorem 1.4 is that the case $\mathcal{W}(X)$ of positive dimension when $X$ is not a cone depends only on the locus $\mathcal{D}(X)$.

Proposition 1.6 Let $X$ be an irreducible, reduced hypersurface in $\mathbb{P}^{n+1}$ which is not a cone. Then $\mathcal{W}(X) \backslash \mathcal{D}(X)$ is finite.

Notice that for all hypersurfaces $X$ having prime degree, the locus $\mathcal{D}(X)$ is empty. These results serve as evidence of the following:

Conjecture 1.7 Let $X$ be an irreducible, reduced hypersurface of $\mathbb{P}^{n+1}, n \geq 1$. Then, the locus $\mathcal{W}(X)$ is finite unless $X$ is a cone.

Plan of the paper In Sect. 2, we recall some basic definitions which will be useful in the following. The theory of focal loci, which is the main technical tool used in this paper, is introduced in Sect. 3. The main results in this context are Lemma 3.7 and Lemma 3.8. The first part of Sect. 4 is devoted to the proof of Theorem 1.2 about non-uniform points, followed by the consequences concerning general projections of smooth varieties. The second part contains the study of families of simply tangent lines passing through $\mathcal{W}(X)$ (see Theorem 1.4) and their relationship with $\mathcal{G}(X)$ and $\mathcal{D}(X)$. We conclude the paper by summarising our results in light of Conjecture 1.7, this is done in Remark 4.11.

Notation From now on, the varieties are assumed to be complex and projective. Let $\mathscr{F}$ be a family of objects parametrised by a scheme $V$. We say the general element of $\mathscr{F}$ satisfies a certain property $P$ if $P$ holds for every element in a Zariski dense open subset of $V$. We will use the notation $\mathbb{G}\left(r, \mathbb{P}^{n}\right)$ for the Grassmannian parametrising linear spaces of projective dimension $r$ contained in $\mathbb{P}^{n}$. We denote by $X^{\text {sing }}$ the singular locus of a variety $X$ and by $X^{\text {sm }}$ its complement. In the following, we will use integral instead of the equivalent notion of irreducible and reduced.

\section{Preliminaries}

In this section, we will briefly recall some results useful for various proofs in Sect. 4. 


\subsection{Monodromy}

We can define the monodromy group of a finite dominant morphism $f: X \rightarrow Y$ of degree $d>1$ between complex integral hypersurfaces in $\mathbb{P}^{n+1}$ as follows. Let $U \subset Y$ be a Zariski open set over which $f$ is étale, and let $y$ denote a point in $U$. We have a well-defined map

$$
\mu: \pi_{1}(U, y) \rightarrow \operatorname{Aut}\left(f^{-1}(y)\right) .
$$

The image $M(f):=\mu\left(\pi_{1}(U, y)\right)$ is called monodromy group of the map $f$; it is a transitive subgroup of $\operatorname{Aut}\left(f^{-1}(y)\right) \simeq S_{d}$.

We can also describe this group by means of Galois extensions: let $K$ be the Galois closure of the extension $k(X) / k(Y)$, where $k(X), k(Y)$ define the fields of rational functions of $X$ and $Y$, respectively. Define the Galois group $G(f)$ of the map $f$ to be the Galois group of the field extension $K / k(Y)$. It turns out that $G(f)$ is isomorphic to $M(f)$, see [16, Section I]. It follows that $M(f)$ does not depend on the choices of $U$ and $y$.

\subsection{Projections}

Let $X \subset \mathbb{P}^{n+c}$ be a smooth variety of dimension $n$. Let $T$ be a linear subspace of dimension $t \leq c-1$ such that $T \cap X=\emptyset$. Fix a target space $\mathbb{P}^{n+c-(t+1)}$ not intersecting $T$, and consider the finite map defined by the linear projection $\pi_{T}: X \rightarrow \mathbb{P}^{n+c-(t+1)}$. Here and in the following, we will always assume the target space to be fixed, thus speaking of the linear projection $\pi_{T}$ instead of $a$ linear projection $\pi_{T}$. The following theorem is the algebraic version of a result of Mather in [21], which provides a powerful tool in order to understand singularities arising from projections. We will apply it in Proposition 4.7 and 4.8, for $t=c-2$ and $t=c-1$, respectively.

Theorem 2.1 [2, Theorem 1] Let $X$ and $T$ as above. For any $i_{1} \leq t+1$, define $\quad X_{i_{1}}:=\left\{x \in X \mid \operatorname{dim}\left(T_{x} X \cap T\right)=i_{1}-1\right\}$. When $X_{i_{1}}$ is smooth, define $X_{i_{1}, i_{2}}:=\left\{x \in X_{i_{1}} \mid \operatorname{dim}\left(T_{x} X_{i_{1}} \cap T\right)=i_{2}-1\right\}$ and so on. When possible, define $X_{i_{1}, \cdots, i_{k}}$ for $i_{k} \leq \cdots \leq i_{2} \leq i_{1}$. For $T$ general, every $X_{i_{1}, \ldots, i_{k}}$ is smooth and, when not empty, its codimension is positive, see [3, Theorem 2] for details. In particular, the codimension of $X_{i_{1}}$ in $X$ is $i_{1} \cdot\left(i_{1}+1\right)$.

We recall here the definition of projective cone and the statement of Bertini's Theorem.

Definition $2.2\left[18\right.$, Ex 3.1] Let $\Gamma \cong \mathbb{P}^{k}$ be a linear subspace in $\mathbb{P}^{n+1}$ and let $Y \subset \Gamma$ be a variety. Let $V \cong \mathbb{P}^{n-k}$ be a linear subspace disjoint from $\Gamma$. The cone over $Y$ of vertex $V$ is a variety in $\mathbb{P}^{n+1}$ defined as the union of the lines joining the vertex $V$ with points of $Y$.

The notion of cone will be used in Lemma 3.8 and Theorem 1.4 in conjunction with the notion of dual variety. An introduction to this topic can be found in [28].

Theorem 2.3 [20, Theorem 3.3.1] Let $X$ be an irreducible variety and $f: X \rightarrow \mathbb{P}^{r}$ a morphism. Fix an integer $d<\operatorname{dim} \overline{f(X)}$. If $L \subset \mathbb{P}^{r}$ is a general $(r-d)$-plane, then $f^{-1}(L)$ is irreducible. 
When dealing with a projective hypersurface $X$, the map $f$ as defined in Theorem 2.3 above is the inclusion $X \hookrightarrow \mathbb{P}^{n+1}$, so we just have $d<\operatorname{dim} X$, and $f^{-1}(L)=X \cap L$. Moreover, by following the proof, it is easy to see that the hypothesis general concerning $L$ means that we can fix a $(r-d-1)$-plane transverse to $X$, and then choose $L$ general among the $(r-d)$-planes containing $L$.

For what concerns the monodromy group, we have the following version of Bertini-type theorem.

Lemma 2.4 Let $X$ be an irreducible variety in $\mathbb{P}^{n+1}$ of dimension $n \geq 2$. Let $H$ be a general linear subspace of codimension $k \geq 1$, and $X_{H}$ the section of $X$ cut by $H$. Then, for a point $P \in H$ such that $P \notin X$, we have

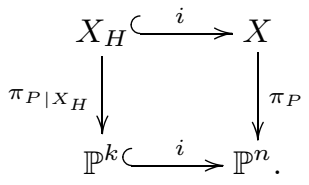

As a consequence, we have $M\left(\pi_{P \mid X_{H}}\right) \leq M\left(\pi_{P}\right)$, which in term of non-uniform points gives that

$\mathcal{W}(X) \cap H \subseteq \mathcal{W}(X \cap H)$

\subsection{Families of tangent lines}

This section is devoted to the study of families of lines that are tangent to a hypersurface. We will briefly go through some preliminaries to highlight the aspects related to our problem; for a more general introduction see for instance [31].

Consider an integral hypersurface $X \subset \mathbb{P}^{n+1}$ of degree $d$ and a line $\ell \nsubseteq X$. The intersection $X \cap \ell$ consists of a finite number of points $P_{1}, \ldots, P_{k}$ counted with multiplicities $m_{1}, \ldots, m_{k}$ such that $\sum m_{i}=d$.

We recall some terminology that is useful to characterise the family of lines with respect to the hypersurface.

Definition 2.5 We call the contact order of $\ell$ with $X$ at $P_{i}$ the number $m_{i}-1$, and we denote it by $\operatorname{ord}_{P_{i}}(\ell \cap X)$. The line $\ell$ is transverse to $X$ at $P_{i}$ if $\operatorname{ord}_{P_{i}}(\ell \cap X)=0$, and tangent to $X$ at $P_{i}$ if $\operatorname{ord}_{P_{i}}(\ell \cap X) \geq 1$. In the case of higher contact order, i.e. $\operatorname{ord}_{P_{i}}(\ell \cap X) \geq 2$, we say that the line $\ell$ is an asymptotic tangent to $X$ at $P_{i}$. The line $\ell$ is called bitangent to $X$ at two points $P_{i} \neq P_{j}$, if $\ell$ is tangent to $X$ at both points $P_{i}, P_{j}$. We say that $\ell$ is a simple tangent if there is a unique tangent point $P_{i} \in \ell \cap X$ with $\operatorname{ord}_{P_{i}}(\ell \cap X)=1$ and $\ell$ is transverse to $X$ for all the other $P_{j} \neq P_{i}$ in $\ell \cap X$.

Notice that if we take a singular point in a hypersurface $X$, then all the lines passing through it will be at least simply tangent. We remark that this is not true when $X$ is a variety of codimension higher than 1 . 
Definition 2.6 [18, Lecture 20] Consider a hypersurface $X$ and a point $P \in X$. Choose an affine neighbourhood of $P$, where $P$ is the origin. In this neighbourhood, $X$ is described by a certain polynomial $f:=f_{m}+f_{m+1}+\cdots$, where $f_{k}$ is homogeneous of degree $k$, and $m$ is the smallest integer such that $f_{m}$ is not vanishing. The tangent cone to $X$ at the point $P$ is the hypersurface described by the polynomial $f_{m}$.

\subsection{Branch locus}

Let $X$ be an integral hypersurface in $\mathbb{P}^{n+1}$ of degree $d$, and $\pi_{P}: X \rightarrow \mathbb{P}^{n}$ be the projection from a point $P \notin X$ and let $y$ be a point in $\mathbb{P}^{n}$. The fibre over $y$ is defined by the set $\pi_{P}^{-1}(y)=\left\{P_{1}, \ldots, P_{k}\right\}$ consisting of $k \leq d$ distinct points. This set corresponds to the settheoretical intersection of $X$ with the line through $P$ and $y$.

Definition 2.7 We call classical branch locus of $\pi_{P}$ the locus $\mathcal{B}$ of points $y \in \mathbb{P}^{n}$ such that the cardinality of the fibre $\pi_{P}^{-1}(y)$ is strictly lower than $d$.

The image via $\pi_{P}$ of $X^{\text {sing }}$ is contained in the classical branch locus, since any line passing through $X^{\text {sing }}$ is tangent to $X$. We want to distinguish the points in the classical branch locus related to $X^{\text {sing }}$ from the points coming from lines in $\mathbb{P}^{n+1}$ for which the tangency order is greater than the order of the general line in $\mathbb{P}^{n+1}$. To this end, consider the normalisation map $v: \tilde{X} \rightarrow X$, and its composition $\phi$ with the projection $\pi_{P}$ :

$$
\phi=\pi_{P} \circ v: \tilde{X} \rightarrow \mathbb{P}^{n} .
$$

Let $y$ be a general point of an irreducible component of $\mathcal{B}$ and let $l$ be the 0 dimensional subscheme of $X$ obtained by cutting $X$ with the line $\langle P, y\rangle$. Thus, its pullback to $\tilde{X}$ is given by $\tilde{l}=m_{1} x_{1}+\cdots+m_{t} x_{t}$, where $d \geq t \geq k$ and $\sum_{i=1}^{t} m_{i}=d$. The image of the singular locus of $\tilde{X}$ via $\phi$ is in codimension 2 in $\mathbb{P}^{n}$. As $y$ is chosen to be general, the points $x_{1}, \ldots, x_{t}$ are smooth in $\tilde{X}$.

Definition 2.8 An irreducible component of $\mathcal{B}$ is called a branch component if the fibre on $\tilde{X}$ of a general point $y$ has at least a (necessarily smooth) point $x_{i}$ with $m_{i} \geq 2$. The union of all the branch components is called the branch locus of $\pi_{P}$. We will denote it by $B_{P}$. Moreover, we can define the branching weight of a point $y$ in the branch locus as

$$
b(y):=\sum_{i=1}^{t}\left(m_{i}-1\right) \geq 1 .
$$

We say that $y \in B_{P}$ is a simple branch point if $b(y)=1$.

There is a relationship between the branching weight and the permutation type of the corresponding element in $S_{d}$ via the monodromy map. In particular, simple branch points correspond to transpositions in the monodromy group, see [16, Section II.3]. The following lemma is proved in [22, Chapter III, Lemma 4.6]. We report its statement in the particular case of projections by using the notation introduced before. 
Lemma 2.9 Consider the projection $\pi_{P}$ of an integral planar curve $X$ from a point $P \notin X$. Let $y$ be a point of $B_{P}$. The cycle structure of the permutation representing a small loop around $y$ in the monodromy group is $\left(m_{1}, \ldots, m_{t}\right)$, where the $m_{i}$ are defined as above.

As a consequence, we can rephrase algebraic results about symmetric groups in terms of tangency and monodromy. For instance, we know that a transitive subgroup of $S_{d}$ which is generated by transpositions, coincides with $S_{d}$. As a consequence, assume $\operatorname{dim} X>1$; if there is a simple tangent line to $X$ passing through $P \in X^{\mathrm{sm}}$, then the general line tangent to $X$ in $P$ is simply tangent. If we take a general hyperplane $H$ passing through $P$, then the point $P \in H$ is uniform for $X \cap H$, and so by Lemma $2.4 P$ is also uniform for $X$.

\section{Focal loci of a family of lines}

In this section, we discuss some special families of lines related to our hypersurface which will be important for their relationship with the monodromy group. We refer the reader to [19, Chapter III.9] and to [27, Chapter 4.6.7] for background material about families of algebraic spaces.

Let $\mathcal{X}$ be a flat family of closed subschemes of $\mathbb{P}^{n+1}$ parametrised by a integral base scheme $S$. This can be described by the following diagram, where the map $i$ is the inclusion and $p, q$ are the projections on the first and second factor, respectively:

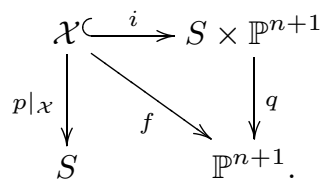

Definition 3.1 The kernel of the differential $\mathrm{d} f$ defines a sheaf $\mathcal{F}$ over $\mathcal{X}$ and it is called focal sheaf. The locus $\mathcal{F}(\mathcal{X})$, i.e. the support of the sheaf, is called the focal scheme or, more classically, focal locus.

From now on, as in [6, Section 4.2], we will consider only the case in which $\mathcal{X}$ is a family of lines in $\mathbb{P}^{n+1}$, for instance by choosing $S$ to be a subscheme of the Grassmannian $\mathbb{G}\left(1, \mathbb{P}^{n+1}\right)$. The family $\mathcal{X}$ can be interpreted via the map $f$ as the subset of the points in $\mathbb{P}^{n+1}$ of the corresponding lines.

Definition 3.2 The family $\mathcal{X}$ of lines in $\mathbb{P}^{n+1}$ over the base $S$ is called filling family if the dimension of $S$ is $n$ and the map $f=i \circ q$ is dominant.

The focal locus $\mathcal{F}\left(\ell_{s}\right)$ restricted to a general line $\ell_{s}$ of the family $\mathcal{X}$ is where the following map has not maximal rank

$$
\mathcal{O}_{\ell_{s}}^{\oplus n} \cong T_{S, s} \otimes \mathcal{O}_{\ell_{s}} \rightarrow \mathcal{N}_{\ell_{s} \mid \mathbb{P} n+1} \cong \mathcal{O}_{\ell_{s}}(1)^{\oplus n}
$$


We will now introduce the notation for proving Lemma 3.5. Let $\mathcal{X}$ be a filling family of lines in $\mathbb{P}^{n+1}$, so that $\operatorname{dim} \mathcal{X}=n$. Assume $\mathcal{X}$ is locally parametrised by $S:=S\left(u_{1}, \ldots, u_{n}\right)$. The line $\ell_{s}$ corresponding to a point $s \in S$ can be described by the intersection of $n$ distinct hyperplanes

$$
\ell_{s}:=\left\{a_{1}(s) \cdot \underline{x}=\cdots=a_{n}(s) \cdot \underline{x}=0\right\} .
$$

Here, $\quad x=\left(x_{0}: \ldots: x_{n+1}\right)$ is the vector of the coordinates in $\mathbb{P}^{n+1}$ and $a_{i}(s)=\left(a_{i}(s)_{0}: \ldots: a_{i}(s)_{n+1}\right)$ determines the i-th hyperplane. We will denote by $\partial_{u_{k}} a_{i}(s)_{j}$ the partial derivative of $a_{i}(s)_{j}$ with respect to the variable $u_{k}$, and inductively for high order derivatives $\partial_{u_{k}, u_{l}} a_{i}(s)_{j}$, and so on. In the following, we will omit the dependency on $s$, by writing for instance just $\partial_{u_{i}} a_{i}$ for the vector $\left(\partial_{u_{i}} a_{1}(s)_{0}: \ldots: \partial_{u_{i}} a_{i}(s)_{n+1}\right)$. With this notation, the equation of the focal locus on the line $\ell_{s}$ is

$$
\operatorname{det}\left(\begin{array}{ccc}
\left(\partial_{u_{1}} a_{1}\right) \cdot \underline{x} & \cdots & \left(\partial_{u_{n}} a_{1}\right) \cdot \underline{x} \\
\vdots & & \vdots \\
\left(\partial_{u_{1}} a_{n}\right) \cdot \underline{x} & \cdots & \left(\partial_{u_{n}} a_{n}\right) \cdot \underline{x}
\end{array}\right)=0,
$$

together with the equations in (1).

We have sketched the proof of the following lemma, which was originally stated in [26]. Our sketch is based on [6, Proposition 4.1].

Lemma 3.3 Let $\mathcal{X}$ be a filling family of lines in $\mathbb{P}^{n+1}$ and let $s \in S$ be a general point of the base. Then the focal locus in the fibre $\ell_{s}$ consists of $n$ points counted with the right multiplicity as roots of Eq. (2).

Definition 3.4 A point $P$ in $\mathbb{P}^{n+1}$ is called fundamental for the family $\mathcal{X}$ if there is a subfamily $\mathscr{X}^{\prime}$ of lines all passing through it. The fundamental locus is the subset of $\mathbb{P}^{n+1}$ of fundamental points.

The following facts on fundamental points are well known, and their origins date back to Segre, in the work [26]:

Lemma 3.5 Consider a filling family of lines in $\mathbb{P}^{n+1}$ and assume there is a subfamily $\mathscr{X}^{\prime}$ of lines all passing through a point $P$. If the dimension of the base of $\mathscr{X}^{\prime}$ is $k$, then $P$ is a focus of multiplicity $k$.

The number of lines of a family $\mathcal{X}$ through a general point of $\mathrm{P}^{n+1}$ is classically called the order of the family $\mathcal{X}$.

Proposition 3.6 [8, Proposition 4.3] Let $\mathcal{X}$ be a filling family of lines in $\mathbb{P}^{n+1}$ of order 1. Then, the focal locus coincides with the fundamental locus.

We now prove two results on the fundamental locus of particular filling families of lines in $\mathbb{P}^{n+1}$ that we will use later. The following result is a generalisation of parts (a) and (c) of Proposition 5.1 in [6].

Lemma 3.7 Consider a filling family $\mathcal{X}$ of lines in $\mathbb{P}^{n+1}$ and an integral hypersurface $X$. Assume the general $\ell \in \mathcal{X}$ is tangent to $X$ at a general point $P$. Then $P$ is a focus on $\ell$. 
Moreover, if the contact order of $\ell$ with $X$ at $P$ is at least 2, then $P$ is a focus with multiplicity at least 2 on $\ell$.

Proof We can assume that the hypersurface $X$ is parametrised locally around $P$ by the same $S$ which parametrises the family $\mathcal{X}$, so $P:=P(s)$ will denote the point varying on $X$, and $\ell_{s}:=\left\{a_{1}(s) \cdot x=\cdots=a_{n}(s) \cdot \underline{x}=0\right\}$ will denote the line tangent at $P(s)$ varying in $\mathcal{X}$, as in Eq. (1). Let $\bar{a}_{1}(s) \cdot \underline{x}=0$ be the hyperplane tangent to $X$ at $P(s)$, so that

$$
a_{1}(s) \cdot P(s)=a_{1}(s) \cdot\left(\partial_{u_{1}} P(s)\right)=\cdots=a_{1}(s) \cdot\left(\partial_{u_{n}} P(s)\right)=0 .
$$

By taking partial derivatives, we get

$$
\left(\partial_{u_{1}} a_{1}(s)\right) \cdot P(s)=\cdots=\left(\partial_{u_{n}} a_{1}(s)\right) \cdot P(s)=0 .
$$

It immediately follows that $P(s)$ satisfies Equation (2), and so is a focus on $\ell(s)$ independently of the choices of the other $n-1$ hyperplanes.

For the second part, since $\ell_{s}$ is tangent to $X$ at $P$, we can assume $\ell$ to be parametrised as follows:

$$
\ell_{s}:=\left\{P(s)+\lambda \partial_{u_{1}} P(s)\right\} .
$$

With this notation, Eq. (2) becomes an equation in $\lambda$ with a parameter $s$. Proving that $P(s)$ is a focus of with multiplicity at least 2 on $\ell_{s}$ means that we can collect $\lambda^{2}$ in the previous equation. The hypothesis that the contact order of $\ell_{s}$ with $X$ at $P(s)$ is at least 2 gives $a_{1}(s) \cdot\left(\partial_{u_{1}, u_{1}} P(s)\right)=0$, and as a consequence $\partial_{u_{1}} a_{1}(s) \cdot\left(\partial_{u_{1}} P(s)\right)=0$. Note that $a_{2}(s), \ldots, a_{n}(s)$ can be choose to be general hyperplanes containing $\ell_{s}$. We have $a_{i}(s) \cdot P(s)=a_{i}(s) \cdot \partial_{u_{1}} P(s)=0$, hence $\partial_{u_{1}} a_{i}(s) \cdot P(s)=0$.

After omitting the dependencies on the point $s \in S$ for better reading, these computations show that the matrix in Eq. (2) becomes

$$
\operatorname{det}\left(\begin{array}{cccc}
0 & \lambda\left(\partial_{u_{2}} a_{1}\right) \cdot\left(\partial_{u_{1}} P\right) & \cdots & \lambda\left(\partial_{u_{n}} a_{1}\right) \cdot\left(\partial_{u_{1}} P\right) \\
\lambda\left(\partial_{u_{1}} a_{2}\right) \cdot\left(\partial_{u_{1}} P\right) & \cdots & \cdots & \cdots \\
\vdots & & & \vdots \\
\lambda\left(\partial_{u_{1}} a_{n}\right) \cdot\left(\partial_{u_{1}} P\right) & \cdots & \cdots & \cdots
\end{array}\right)=0 .
$$

Lemma 3.3 guarantees that this determinant is not identically zero whenever $\ell_{s}$ is a general element of the family. Such a determinant is a multiple of $\lambda^{2}$, hence the point $P$ is a focus of multiplicity at least 2 .

In the proof of Theorem 1.2, we will have to deal also with a family of lines obtained by joining a curve and a variety of codimension 2 . At this purpose, we generalise $[8$, Lemma 2] to higher dimension.

Lemma 3.8 Let $F$ be a subvariety of $\mathbb{P}^{n+1}$ of codimension 2, and $\mathscr{C} \nsubseteq F$ be a curve not contained in a $\mathbb{P}^{n-1}$. Assume that the family $\mathcal{X}$ of lines joining $\mathscr{C}$ and $F$ is filling. Then, $F$ is linear and $\mathscr{C}$ is rational. If $\mathscr{C} \cap F=\emptyset, \mathscr{C}$ is also linear. Otherwise, $F$ meets $\mathscr{C}$ in $\operatorname{deg}(\mathscr{C})-1$ points.

Proof In this proof we will follow [8]. We also refer to it for the notations and preliminaries about Schubert cycles. The points of $F$ and the points of $\mathscr{C}$ are fundamental, they yield 
on the general $\ell \in \mathcal{X}$ a focus of multiplicity $n-1$ in $\ell \cap C$ and a focus of multiplicity 1 in $\ell \cap F$. By Lemma 3.3 there are no other focal points on $\ell$, and so the focal locus has codimension exactly 2 . We can apply [9, Theorem 2.1] and conclude that the order of $\mathcal{X}$ must be 1 since we are assuming $\mathcal{X}$ is filling. Let $d_{1}$ and $d_{2}$ be the degree of $\mathscr{C}$ and $F$, respectively. Assume first that $\mathscr{C} \cap F \neq \emptyset$, and let $m$ be the number of lines through a general point $P \in \mathbb{P}^{n+1}$ passing through $\mathscr{C} \cap F$. The cone $\chi_{\mathscr{C}, P}$ over $\mathscr{C}$ of vertex $P$ has dimension 2 and degree $d_{1}$, while the cone $\chi_{F, P}$ has dimension $n$ and degree $d_{2}$. They intersect in $d_{1} d_{2}$ lines through the point $P$, and we know that one of these lines belongs to the family $\mathcal{X}$. Since the family of lines through each point of $\mathscr{C} \cap F$ form itself a filling family of order one, that line does not pass through $\mathscr{C} \cap F$. Therefore, we have that $m=d_{1} d_{2}-1$.

Assume by contradiction that $F$ is not linear. If $F$ is non degenerate, consider the cone $\chi_{F, Q}$, where $Q$ is a general point of $F$, which has degree $d_{2}-1$. Recall that the general line of $\mathcal{X}$ meets $\mathscr{C}$ (resp. $F$ ) at a single point and those are the only focal points on the line. The cone $\chi_{F, Q}$ does not contain the cone $\chi_{\mathscr{C}, Q}$ since otherwise the lines secant to $F$ and intersecting $\mathscr{C}$ will form a hypersurface of focal points.

As before, the intersection of the two cones gives $d_{1}\left(d_{2}-1\right)$ lines through $Q$ meeting $\mathscr{C} \cap F$ : if one of these lines meets $\mathscr{C}$ and $F$ in distinct points, then all the line would be focal, and this contradicts the fact that the focal locus has codimension 2. Therefore, we have that $m=d_{1}\left(d_{2}-1\right)$. Summing up, $d_{2} d_{1}-1=d_{2} d_{1}-d_{1}$ which gives that $d_{1}=\operatorname{deg}(\mathscr{C})=1$ that is a contradiction. If $F$ is contained in a hyperplane $H$, then $\mathscr{C}$ is not contained in $H$ since the family $\mathcal{X}$ is filling. Hence, the number of points in $\mathscr{C} \cap F$ is lesser than or equal to $d_{1}=\operatorname{deg}(\mathscr{C})$. As before, we have that $m=d_{1} d_{2}-1 \leq d_{1}$ and so $\mathscr{C}$ is linear and $F$ has degree 2 , that is again a contradiction. Therefore, $F$ must be linear.

We are left with the case $\mathscr{C} \cap F=\emptyset$. By following the notation of [8], let $M(F)$ be the family of lines in $\mathbb{P}^{n+1}$ meeting $F$. It is a codimension 1 family in $\mathbb{G}\left(1, \mathbb{P}^{n+1}\right)$, hence it can be written as

$$
M(F)=d_{2} \sigma_{1,0}
$$

In the same way, let $M(\mathscr{C})$ be the family of lines meeting $\mathscr{C}$. It is a codimension $n-1$ family in $\mathbb{G}\left(1, \mathbb{P}^{n+1}\right)$, hence it can be written as

$$
M(\mathscr{C})=\sum_{t=0}^{k} a_{t} \sigma_{n-1-t, t}=a_{0} \sigma_{n-1,0}+\cdots+a_{k} \sigma_{n-1-k, k},
$$

where $k=\left\lfloor\frac{n-1}{2}\right\rfloor$. The complementary cycle of $\sigma_{n-1-t, t}$ with $t>0$ is $\sigma_{n-t, t+1}$, which gives a family of lines contained in a $\mathbb{P}^{n-t}$. But if $t \geq 1$, a general $\mathbb{P}^{n-t}$ does not intersect the curve $\mathscr{C}$, hence we have that $M(\mathscr{C}) \cdot \sigma_{n-t, t+1}=a_{t}=0$ for $t>0$. The complementary cycle of $\sigma_{n-1,0}$ is $\sigma_{n, 1}$, which gives a family of lines contained in a $\mathbb{P}^{n}$ passing through a general point. A general hyperplane cuts $\mathscr{C}$ in $d_{1}$ points and the lines passing through them are lines of $M(\mathscr{C})$ contained in this hyperplane. Hence, $M(\mathscr{C})$ reduces to $d_{1} \sigma_{n-1,0}$. The filling family $\mathcal{X}$ is given by the intersection of $M(F)$ and $M(\mathscr{C})$. Pieri's formula gives

$$
M(F) \cdot M(\mathscr{C})=d_{1} d_{2}\left(\sigma_{n, 0}+\sigma_{n-1,1}\right),
$$

saying that through a general point of $\mathbb{P}^{n+1}$ pass $d_{1} d_{2}$ lines of $\mathcal{X}$. By our assumption, $d_{1}=d_{2}=1$, i.e. both $\mathscr{C}$ and $F$ are linear. The proof of the rationality of $\mathscr{C}$ follows the same lines of [8, Theorem 7]. 


\section{The locus of non-uniform points}

The first part of this section is devoted to the proof of Theorem 1.2. We will apply all the focal machinery developed in the previous section to the following family.

Definition 4.1 Let $\mathcal{V}$ be the family in $\mathbb{G}\left(1, \mathbb{P}^{n+1}\right)$ composed by the lines $\ell$ of the following types:

$\left(C_{1}\right)$ The line $\ell$ if is bitangent or asymptotic tangent to $X^{\mathrm{sm}}$;

$\left(C_{2}\right)$ The line $\ell$ passes through a point of $X^{\text {sing }}$ and is also tangent at a (necessarily different) point of $X^{\mathrm{sm}}$;

$\left(C_{3}\right)$ The line $\ell$ intersects $X^{\text {sing }}$ in more than one point;

$\left(C_{4}\right)$ The line $\ell$ is in the tangent cone to $X$ at a point in $X^{\text {sing }}$, see Definition 2.6.

If $\mathscr{Y}$ is a variety in $\mathbb{P}^{n+1}$ we define $\mathcal{V}_{\mathcal{Y}}$ the subfamily of $\mathcal{V}$ composed of the lines passing through points of $\mathcal{Y}$.

The subfamily of lines of type $\left(C_{k}\right)$ has expected dimension $2 n-2$ for $k=1, \ldots, 4$. The whole family $\mathcal{V}$ is deeply related with non-uniform points. Indeed, if we take $Q \in \mathcal{W}(X)$, the following lemma shows that the family $\mathcal{V}_{Q}$ defined above has the greatest possible dimension. This will be crucial in the proof of Theorem 1.2.

Lemma 4.2 Let $X$ be an integral hypersurface in $\mathbb{P}^{n+1}$, and let $Q \in \mathcal{W}(X)$ be a non-uniform point. Then, the base parametrising the family $\mathcal{V}_{Q}$ has dimension $n-1$ in the Grassmannian $\mathbb{G}(1, n+1)$.

Proof For $n=1$, [25, Proposition 2.5] guarantees that a non-uniform point must have at least two non-simple tangent lines passing through it. We proceed now by induction. Assume that the claim is true for a hypersurface of $\operatorname{dim} X=n-1$ and prove it for the case $\operatorname{dim} X=n$. By contradiction, assume that the dimension of the base of $\mathcal{V}_{Q}$ is smaller than $n-1$. Take a general hyperplane $H$ in $\mathbb{P}^{n+1}$ passing through $Q$; by Bertini's Theorem, the section $X \cap H$ is integral since $Q \notin X$. The hyperplane $H$ meets the family $\mathcal{V}_{Q}$ in a subfamily parametrised by a base of dimension strictly lower than $n-1$, but this contradicts the induction hypothesis.

Lemma 4.3 Let $\mathscr{C} \subset \mathcal{W}(X)$ be an irreducible curve not contained in a linear space of codimension 2. Then the family $\mathcal{V}_{\mathscr{C}}$ is filling.

Proof We want to show that $\mathcal{V}_{\mathscr{C}}$ is a family whose base space has dimension $n$ and the map $\mathcal{V}_{\mathscr{C}} \rightarrow \mathbb{P}^{n+1}$ of Definition 3.2 is dominant. For every choice of $Q \in \mathscr{C}$, the dimension of the base of $\mathcal{V}_{Q}$ is $n-1$ thanks to Lemma 4.2. Every line in $\mathcal{V}_{\mathscr{C}}$ belongs to the cone $\mathcal{V}_{Q}$ for a certain $Q \in \mathscr{C}$, so the dimension of the base of $\mathcal{V}_{\mathscr{C}}$ is $n$.

If the map $\mathcal{V}_{\mathscr{C}} \rightarrow \mathbb{P}^{n+1}$ of Definition 3.2 were not dominant, then the union of all the $\mathcal{V}_{Q}$ would be contained a finite union of divisors $V_{j}, j=1, \ldots, r$ in $\mathbb{P}^{n+1}$. For a general $Q \in \mathscr{C}$, $\mathcal{V}_{Q}$ is the union of cones over $V_{j} \cap X$ with vertex $Q$, each of which correspond to $V_{j}$ since they have the same dimension, again by Lemma 4.2. Let us work with just one $V_{j}:=V$ for some $j=1, \ldots, r$. The cone $\mathcal{V}_{Q}$ coincides with $\mathcal{V}_{Q^{\prime}}$ for every $Q, Q^{\prime} \in \mathscr{C}$ and we will just denote it by $V$. We claim that $V$ is a linear space. Indeed, since $\mathscr{C}$ is contained in the vertex 
of $V$, then we can apply [1, Proposition 1.3 (i)] and get that also the linear span $\langle\mathscr{C}\rangle$ must be contained in the vertex of $V$. From the assumption on the curve, $V$ must be linear.

As a consequence, the curve $\mathscr{C}$ must be contained in the intersection of $H_{1}, \ldots, H_{k}$, so we have to rule out the case in which $k=1$. A general $\mathbb{P}^{2}$ passing through a general point $Q$ of $\mathscr{C}$ will intersect $X$ in an integral curve. The hyperplanes $H_{i}$ intersect this last $\mathbb{P}^{2}$ in lines which, together with some transposition coming from lines outside the $H_{i}$ 's, are generators of the monodromy group $\pi_{Q}$. Recall that the product of all the generators is the identity: if $k=1$, the monodromy group $\pi_{Q}$ will be generated just by the transpositions outside $H_{1}$. However, this cannot happen, as the point $Q$ is not uniform. Consequently, there must be at least two generators coming from the $H_{i}$, hence $k>1$.

The problem of finding a bound on the dimension of $\mathcal{W}(X)$ for planar curves has been completely solved in [25]:

Theorem 4.4 [25, Theorem 3.5, $r=2]$ Let $X \subset \mathbb{P}^{2}$ be an irreducible curve. Then the locus of non-uniform points is finite.

Notice that by subsequently taking general hyperplane sections (see Lemma 2.4), Theorem 4.4 implies that the codimension of $\mathcal{W}(X)$ must be at least 2 . In order to prove the main theorem, we start by the following lemma, in which under some technical hypotheses, we use the theory of focal loci to get a crucial property of certain curves contained in $\mathcal{W}(X)$ which will be useful in the following.

Lemma 4.5 Let $X$ be an integral hypersurface of $\mathbb{P}^{n+1}, n \geq 2$. Assume $\mathscr{C} \subset \mathcal{W}(X)$ is a curve such that $\mathcal{V}_{\mathscr{C}}$ has a component of maximal dimension of lines of type $\left(C_{1}\right),\left(C_{2}\right)$ or $\left(C_{3}\right)$. Then, $\mathscr{C}$ must be contained in a linear space of codimension 2 in $\mathbb{P}^{n+1}$.

Proof If the thesis is false, we can assume $\mathscr{C}$ is not contained in a linear space of codimension 2 . We want to apply the focal machinery to the family $\mathcal{V}_{\mathscr{C}}$. The hypothesis of Lemma 4.3 are satisfied, so we know that $\mathcal{V}_{\mathscr{C}}$ is filling. Consider a general element $\ell \in \mathcal{V}_{\mathscr{C}}$.

The general element of $\mathcal{V}_{\mathscr{C}}$ is of type $\left(C_{1}\right)$. We claim that the general $\ell$ of $\mathcal{V}_{\mathscr{C}}$ is tangent at a general point of $X$. Indeed, if it were not true, there would be a divisor $Y \subset X^{\mathrm{sm}}$ such that the family $\mathcal{V}_{\mathscr{C}}$ of lines would be the join of $Y$ and $\mathscr{C}$. Therefore, $\mathscr{C}$ would be contained in the tangent hyperplane $T_{y} X$ for every $y \in Y$, so $\mathscr{C}$ belongs to the intersection of all the $T_{y} X$, and the lines of $\mathcal{V}_{\mathscr{C}}$ belong to the union of all the $T_{y} X$. Since we assumed $\mathscr{C}$ not be contained in a linear space of codimension 2, the only possibility is that that $T:=T_{y} X$ is constant for $y \in Y$. But then $\mathcal{V}_{\mathscr{C}}$ would be contained in $T$ hence not filling, contradicting Lemma 4.3.

If $\ell$ is an asymptotic tangent line at a general point $P \in X$, then it is a focal point with multiplicity 2 ; if $\ell$ is bitangent at two distinct points then both of them are focal points for $\ell$ (see Lemma 3.7).

The general element of $\mathcal{V}_{\mathscr{C}}$ is of type $\left(C_{2}\right)$.

As for the previous case, we can assume that the point in $\ell$ is tangent at a general point in $X^{\mathrm{sm}}$, so the tangency point is a focus by Lemma 3.7, while a point in $\ell \cap X^{\text {sing }}$ is focal by Lemma 3.5.

The general element of $\mathcal{V}_{\mathscr{C}}$ is of type $\left(C_{3}\right)$. The family $\mathcal{V}_{\mathscr{C}}$ consists of lines passing through at least two points of $X^{\text {sing }}$ and intersecting $\mathcal{W}(X)$. Hence, there is a 
one-dimensional subfamily of lines of $\mathcal{V}_{\mathscr{C}}$ through every point in $X^{\text {sing }}$. By Lemma 3.5, the points in $\ell \cap X^{\text {sing }}$ are focal points for $\ell$, each of multiplicity 1 .

In each of the previous cases, the focal locus of $\ell$ has multiplicity at least 2 in points where $\ell$ is tangent to $X$. Moreover, $\ell$ passes through a point of $\mathscr{C}$ and, by construction, for every such point there is a $n-1$ dimensional subfamily of $\mathcal{V}_{\mathscr{C}}$. Therefore, this point is a focus for $\ell$ as well, its multiplicity being, at least, $n-1$ by Lemma 3.5. Note that the general line meets $\mathscr{C}$ in a point outside $X$. Thus, we have at least $n+1$ focal points in a general line $\ell$ of the filling family $\mathcal{V}_{\mathscr{C}}$. But this is a contradiction because of Lemma 3.3, as the focal locus in a general line of a filling family of lines in $\mathbb{P}^{n+1}$ consists of $n$ points counted with multiplicity.

We are now ready for the proof of the main result of this paper.

Proof of Theorem 1.2 Let us assume that there exists a component of $\mathcal{W}(X)$ not contained in a linear space of codimension 2. Consider an irreducible curve $\mathscr{C} \subset \mathcal{W}(X)$ with the same property. If $\mathcal{V}_{\mathscr{C}}$ has a component of maximal dimension of lines of type $\left(C_{1}\right),\left(C_{2}\right)$ or $\left(C_{3}\right)$, we apply Lemma 4.5 and get a contradiction. We are left to consider the case in which the general $\ell \in \mathcal{V}_{\mathscr{C}}$ is of type $\left(C_{4}\right)$. In this case, $\ell$ belongs to the tangent cone to $X$ at a point $x$ in $X^{\text {sing }}$. As a consequence, $\mathscr{C}$ must be contained in the intersection of all the tangent cones at points in $X^{\text {sing }}$. The family $\mathcal{V}_{\mathscr{C}}$ is composed by lines joining $\mathscr{C}$ and $X^{\text {sing }}$. Recall that we are still assuming $\mathscr{C}$ not contained in a linear space of codimension 2 , hence $\mathcal{V}_{\mathscr{C}}$ is filling and, by applying Lemma 3.8, we get that $\mathscr{C}$ is rational, $X^{\text {sing }}$ is linear and meets $\mathscr{C}$ in $\operatorname{deg}(\mathscr{C})-1$ points. There could be only a finite number of curves $\mathscr{C} \subset \mathcal{W}(X)$ satisfying these properties. Therefore, the dimension of $\mathcal{W}(X)$ must be at most 1 . We also remark that the image of $X^{\text {sing }}$ under the projection from a point is a linear subspace of $\mathbb{P}^{n}$ and, by Lemma 2.9 gives generators of the monodromy group of the projection which are not transpositions. We are assuming the general $\ell \in \mathcal{V}_{\mathscr{C}}$ is of type $\left(C_{4}\right)$, hence $X^{\text {sing }}$ must split in at least two linear spaces, in order to have a non-uniform monodromy.

We remark that the only obstruction to prove that $\mathcal{W}(X)$ is always contained in a linear space of codimension 2 comes from the general $\ell \in \mathcal{V}_{\mathscr{C}}$ being of type $\left(C_{4}\right)$. Notice that the case of $\mathcal{W}(X)$ being a finite union of linear space of codimension 2 can actually happen, as shown in the following example.

Example 4.6 Fix an integral curve $C \subset \mathbb{P}^{n+1}$ contained in a plane $H \cong \mathbb{P}^{2}$ and consider a linear space $V$ of dimension $n-2$ disjoint from $H$. Let $X$ be the cone on $C$ with vertex $V$. We claim that $\mathcal{W}(X)$ is the join of $\mathcal{W}(C)$ and $V$. Assume that $Q$ is a non-uniform point for $C$ in $H$. First, notice that every point in the line $\langle Q, V\rangle$ is non-uniform for $X$ : the linear projection from the vertex induces an isomorphism from the general $\mathbb{P}^{2}$ to $H$ which sends $X \cap \mathbb{P}^{2}$ to $C$, so $\langle\mathcal{W}(C), V\rangle \subset \mathcal{W}(X)$. In a similar way, if $Q \in \mathcal{W}(X)$ we have that $\langle Q, V\rangle \cap H$ is a non-uniform point for $C$, hence $\mathcal{W}(X) \subset\langle\mathcal{W}(C), V\rangle$. In a similar flavour, cones provide an example of an integral $X \subset \mathbb{P}^{n+1}$ with $\mathcal{W}(X)$ being a finite union of $\mathbb{P}^{k}, k=1, \ldots, n-1$.

As a consequence of the main theorem, we are able to prove the finiteness of $\mathcal{W}(X)$ for many hypersurfaces. As a generalisation of Theorem 1.1 in [7] we have Theorem 1.3. We prove it more generally by obtaining the analogous of [25, Theorem 3.5] in higher dimension. Consider a smooth, irreducible variety $\tilde{X}$ of dimension $n$ in $\mathbb{P}^{n+c}, c \geq 1$. We 
can define the monodromy group $M\left(\pi_{L}\right)$ associated with the projection from a linear space $L \in \mathbb{G}\left(c-1, \mathbb{P}^{n+c}\right)$. Our next step is to study the dimension of $\mathcal{W}(\tilde{X})$. We start with projecting $\tilde{X}$ to a hypersurface $X \subset \mathbb{P}^{n+1}$, and to study $\mathcal{W}(X)$.

Proposition 4.7 Let $\tilde{X}$ be a smooth irreducible projective variety of dimension $n$ in $\mathbb{P}^{n+c}$ and $X$ the projection of $\tilde{X}$ from a general linear subspace $L \subset \mathbb{P}^{n+c}$ of dimension $c-2$. Then, the locus $\mathcal{W}(X)$ is at most finite.

Proof Assume by contradiction that $\mathcal{W}(X)$ is not finite, and moreover that there exists a component of $\mathcal{W}(X)$ not contained in a linear space of codimension 2. Consider an irreducible curve $\mathscr{C} \subset \mathcal{W}(X)$ with the same property. We start by a remark on the tangent cone at points in $X^{\text {sing }}$. In particular, we claim that the general line of the family $\mathcal{V}_{\mathscr{C}}$ is not of type $\left(C_{4}\right)$. If $X^{\text {sing }}$ has codimension greater than 1 , this is trivially true. Assume now $X^{\text {sing }}$ of codimension 1. A point $\tilde{X} \in \tilde{X}$ is projected to $x \in X^{\text {sing }}$ in two cases: either the $\mathbb{P}^{c-1}$ given by the join $\langle L, x\rangle$ is secant to $\tilde{X}$ in other points $\tilde{y}_{1}, \ldots \tilde{y}_{k}$, or $x$ belongs to the locus $X_{t}$ described in Theorem 2.1 for a certain $t \geq 1$. In the first case, the tangent cone to $X$ at the point $x$ is a finite union of hyperplanes, corresponding to the projection of the tangent spaces to $\tilde{X}$ at $\tilde{x}, \tilde{y}_{1}, \ldots \tilde{y}_{k}$. The locus of points on $X$ corresponding to the second case has codimension greater than 1 thanks to Theorem 2.1. As a consequence, the general element of $\mathcal{V}_{\mathscr{C}}$ is of type $\left(C_{1}\right),\left(C_{2}\right)$ or $\left(C_{3}\right)$ and Lemma 4.5 gives a contradiction. Hence, $\mathcal{W}(X)$ must be contained in a linear space of codimension at least 2 .

Denote by $K \cong \mathbb{P}^{k}$ the smallest linear subspace of $\mathbb{P}^{n+1}$ containing a irreducible component of $\mathcal{W}(X)$ and assume that the curve $\mathscr{C} \subset \mathcal{W}(X)$ spans $K$. Consider a general $H \cong \mathbb{P}^{k+1}$ containing $K$. Since $X$ is reduced, also is $X \cap H$. We will first show that assuming $X \cap H$ to be irreducible leads to a contradiction. By Lemma 4.3, since $\mathscr{C}$ spans a space of codimension 1 in $H$, the family $\mathcal{V}_{\mathscr{C}, H}$ of lines in $\mathcal{V}_{\mathscr{C}}$ which belongs to $H$ is filling. If the hypothesis of Lemma 4.5 is satisfied we immediately get a contradiction. So the only possibility is that the general element of $\mathcal{V}_{\mathscr{C}, H}$ is of type $\left(C_{4}\right)$. This was not true for the family $\mathcal{V}_{\mathscr{C}}$, due to the dimension of the locus in $X^{\text {sing }}$ giving rise to lines of type $\left(C_{4}\right)$. Since $H$ is general, the only possibility is that the general $\ell \in \mathcal{V}_{\mathscr{C}, H}$ is tangent to $X$ at the base locus of $X \cap H$, namely in points of $X \cap K$. This, however, gives that $\ell$ is contained in $K$, contradicting the fact that $\mathcal{V}_{\mathscr{C}, H}$ is filling.

We are left with the case in which $X \cap H$ is reducible. Denote by $\tilde{K}$ and $\tilde{H}$ the linear spaces $\langle L, K\rangle$ and $\langle L, H\rangle$, respectively. The variety $X \cap H$ is the linear projection from $L$ of $\tilde{X} \cap \tilde{H}$, hence also $\tilde{X} \cap \tilde{H}$ must be reducible since the projection is a continuous map. In the case we are considering, Theorem 2.3 cannot be applied, hence $\tilde{K}$ is not transverse to $X$, namely for all $x \in \tilde{X} \cap \tilde{K}$, the tangent space $T_{x} \tilde{X}$ is contained in $\tilde{K}$. The dimension of the intersection of $T_{x} \tilde{X}$ with $L$ in $\tilde{K}$ is then $n-k-1$, which is greater than or equal to 0 , since $1 \leq k \leq n-1$. This shows that $\tilde{X} \cap \tilde{K}$ is contained in the locus $\tilde{X}_{n-k}$ defined in Theorem 2.1. Now, $\tilde{X} \cap \tilde{K}$ has dimension $k-1$ and $\tilde{X}_{n-k}$ has dimension $n-(n-k)(n-k+1)$. We get

$$
\begin{aligned}
k-1 & \leq n-(n-k)(n-k+1) \\
0 & \leq 1-(n-k)^{2}
\end{aligned}
$$

The only possibility is $k=n-1$, for which $\tilde{X} \cap \tilde{K}$ has the same dimension of $\tilde{X}_{1}$. We know by Theorem 2.1 , that $\tilde{X}_{1}$ is smooth, and this gives a contradiction. 
As a consequence of this proposition, we can reason as in [25, Theorem 3.5] and show the following

Proposition 4.8 Let $\tilde{X}$ be a smooth irreducible complex projective variety of dimension $n$ in $\mathbb{P}^{n+c}, c \geq 1$. The locus of non-uniform $(c-1)$-planes $L$ not intersecting $\tilde{X}$ has codimension at least $n+1$ in the Grassmannian $\mathbb{G}\left(c-1, \mathbb{P}^{n+c}\right)$.

Proof We will follow the proof of [25, Theorem 3.5]. When $c=1$ we know from Proposition 4.7 that all but finitely many points $P \in \mathbb{P}^{n+1} \backslash \tilde{X}$ are uniform. Now assume $c \geq 2$. After projecting from a general $(c-2)$-subspace $M$, we get $X \subset \mathbb{P}_{M}^{n+1}$, where $\mathbb{P}_{M}^{n+1}$ parametrises all the $(c-1)$-planes $L$ containing $M$. Notice that projecting $\tilde{X}$ to $\mathbb{P}^{n}$ from $L$ is equivalent to projecting $X$ to $\mathbb{P}^{n}$ from the point in $\mathbb{P}_{M}^{n+1}$ corresponding to $L$. Proposition 4.7 applied to $X$ gives $\operatorname{dim} \mathcal{W}(X) \leq 0$.

Assume by contradiction that $\mathcal{W}(\tilde{X})$ has codimension at most $n$ in the Grassmannian $\mathbb{G}\left(c-1, \mathbb{P}^{n+c}\right)$. In this case, there would be an irreducible subvariety $D$ of codimension at most $n$ such that the general $L \in D$ is non-uniform, i.e. every $L \in D \backslash Z$ is non-uniform for a proper Zariski closed subset $Z$. We claim that for a general element $M \in \mathbb{G}\left(c-2, \mathbb{P}^{n+c}\right)$, the dimension of $(D \backslash Z) \cap \mathbb{P}_{M}^{n+1}$ is greater than zero. Notice first that $D \cap \mathbb{P}_{M}^{n+1}$ is at least one-dimensional: $D$ has codimension at most $n$ in $\mathbb{G}\left(c-1, \mathbb{P}^{n+c}\right)$ and $\mathbb{P}_{M}^{n+1}$ is $n+1$ dimensional. Secondly, we have that

$$
\operatorname{dim}\left(Z \cap \mathbb{P}_{M}^{n+1}\right)<\operatorname{dim}\left(D \cap \mathbb{P}_{M}^{n+1}\right) .
$$

This implies that there exist infinitely many non-uniform such planes $L$ containing a general $M \in \mathbb{G}\left(c-2, \mathbb{P}^{n+c}\right)$, but this would give $\operatorname{dim}(X)>0$, which contradicts Proposition 4.7.

Remark 4.9 The same argument of [25, Remark 3.6] shows that the bound in Proposition 4.8 is sharp. There are singular varieties $X$ for which there exist points $x \notin X$ such that the projection $\pi_{x}: X \subset \mathbb{P}^{n+c} \rightarrow \mathbb{P}^{n+c-1}$ is non-birational onto the image. If this is the case, a $(c-1)$-plane $L$ containing such an $x$ is non-uniform because the map $\pi_{L}$ factorises in a nontrivial way. Thus, the family of the $(c-1)$-planes passing through $x$ is a family consisting of non-uniform elements in $\mathbb{G}\left(c-1, \mathbb{P}^{n+c}\right)$ of codimension $n+1$.

We now focus on the case in which $\mathcal{W}(X)$ is not finite. Transpositions play a fundamental role in determining if a point is uniform or not, see [25, Remark 2.2]. This motivates Theorem 1.4, in which as in Lemma 4.5, we apply Lemma 3.7 to show that if $\operatorname{dim} \mathcal{W}(X)>0$ and $X$ is not a cone, then the monodromy group associated with all but finitely many points of $\mathcal{W}(X)$ contains transpositions.

Proof of Theorem 1.4 Consider the family $\mathcal{X}$ of lines in $\mathbb{P}^{n+1}$ tangent to $X$ at smooth points and passing through a curve $\mathscr{C}$ inside $\mathcal{W}(X)$.

Notice that $\mathcal{X}$ is composed by lines lying on hyperplanes tangent to $X$ and passing through $\mathscr{C}$. Let $X^{*} \subset\left(\mathbb{P}^{n+1}\right)^{*}$ be the dual variety of $X$; let $r$ be the dimension of $X^{*}$. If $X$ is not a cone, by [28, Theorem 1.25] we have that $X^{*}$ is not contained in a hyperplane. Consider the family of hyperplanes in $\left(\mathbb{P}^{n+1}\right)^{*}$ dual to the points of $\mathscr{C}$. The general hyperplane of this family intersects $X^{*}$ in a locus of dimension $r-1$. Moreover, every point of $X^{*}$ is contained in one of such hyperplanes, which dualising means that the tangent hyperplane to $X$ at a general point pass through the general point of $\mathscr{C}$. As a consequence, for the general $Q \in \mathscr{C}$, we have a $r-1$ dimensional family of tangent hyperplanes to $X$. The general 
member of this family is tangent to $X$ along a subvariety of dimension $n-r$. Every line joining $Q$ and this $n-r$ subvariety is tangent to $X$, so in particular the dimension of the family $\mathcal{X}$ is $n$, and the general line in $\mathcal{X}$ is tangent at the general point of $X$.

To show that $\mathcal{X}$ is filling we still have to prove that the map $\mathcal{X} \rightarrow \mathbb{P}^{n+1}$ is dominant. If this were not the case, the general line of $\mathcal{X}$ would be contained in a finite union of hypersurfaces $V_{i}$, hence it would not be tangent at the general point of $X$.

Let us consider the focal scheme of $\mathcal{X}$ on $\ell$. We have a fundamental point $\ell \cap Q$ of multiplicity $n-1$, hence a focus of multiplicity $n-1$ by Lemma 3.5. Assume that the general line $\ell$ of $\mathcal{X}$ is not simply tangent to $X$. Then, it would be at least bitangent or asymptotic tangent to $X$. In the first case, we would have on $\ell$ at least two focal points of degree at least 1 each, in the second case we would have at least one focal point of degree at least 2 . This contradicts Lemma 3.3.

As a consequence, if $X$ is not a cone, we can find simple tangent lines to $X$ passing through all but finitely many points of $\mathcal{W}(X)$. Such lines correspond to transpositions in the monodromy group.

From Theorem 1.4, we get Proposition 1.5, that is a generalisation of [12, Proposition 6] on Galois points.

Proof of Proposition 1.5 Denote by $\mathcal{G}(X)$ the locus of Galois points associated with $X$. Recall that $\mathcal{G}(X) \subset \mathcal{W}(X)$. If we assume $\mathcal{G}(X)$ to be an infinite set and $X$ not a cone, Theorem 1.4 shows the existence of transpositions in the monodromy group associated with a general point $Q$ in $\mathcal{G}(X)$. As a consequence, the field extension given by $\pi_{Q}$ is not Galois, and this contradicts our initial hypothesis.

Recall that if $M\left(\pi_{P}\right)$ is isomorphic to the full symmetric group then the projection $\pi_{P}$ is indecomposable. The converse also holds if we require $M\left(\pi_{P}\right)$ to contain a transposition (see [25, Remark 2.2]). Hence, we have Proposition 1.6.

Proof of Proposition 1.6 By Theorem 1.4, the monodromy group of all but finitely many points contains a transposition. Hence, for all but finitely many points $Q$ in $\mathcal{W}(X)$, the projection $\pi_{Q}$ must be a decomposable map, i.e. the point $Q$ belongs to $\mathcal{D}(X)$.

Remark 4.10 Notice that this is enough to prove Conjecture 1.7 for all hypersurfaces $X$ having prime degree. Indeed, $\pi_{P}: X \rightarrow \mathbb{P}^{n}$ is indecomposable for every $P \notin X$ because otherwise, the degree of an intermediate, not birational map would divide $d$.

There are two classes of hypersurfaces which could potentially provide a counterexample for Conjecture 1.7:

Type-1: Hypersurfaces $X$ in $\mathbb{P}^{n+1}$, where every component of $X^{\text {sing }}$ in codimension 1 is linear, and such that the intersection of all the tangent cones at points in $X^{\text {sing }}$ is a finite union of rational curves.

Type-2: Hypersurfaces $X$ in $\mathbb{P}^{n+1}$ such that there exists a $\mathbb{P}^{k}(0<k<n)$, where $X \cap \mathbb{P}^{k+1}$ is reducible for every $\mathbb{P}^{k+1} \supset \mathbb{P}^{k}$. Cones are a particular case of $X$ of Type-2.

Remark 4.11 We have that if $X$ is neither of Type- 1 nor of Type-2, then $\mathcal{W}(X)$ is finite. The proof mimics the steps of Proposition 4.7: if $X$ is not of Type-1 we can apply Theorem 1.2 to get that that $\mathcal{W}(X) \subset \mathbb{P}^{n-1}$; if $X$ is not of Type-2 either, we can apply Lemma 2.4 . 
While examples of hypersurfaces of Type- 1 and Type- 2 do exist, we were not able to find any variety with an infinite number of non-uniform points that is not a cone. We plan to study these cases in a future work.

Acknowledgements M.G.C. and R.M. are supported by MIUR: Dipartimenti di Eccellenza Program (2018-2022) - Dept. of Math. Univ. of Pavia and by PRIN 2017 "Moduli spaces and Lie Theory" code 2017YRA3LK_003. We are indebted to an anonymous referee for many useful suggestion which improved the exposition and the quality of this paper. We would like to thank Gian Pietro Pirola for introducing us to the problem and the different techniques involved; we also thank him for all the help he gave us during the preparation of this paper. We are also grateful to Ciro Ciliberto for constructive discussions about how to generalise some result concerning focal loci to a higher dimension. We benefit from many helpful discussions with Thomas Dedieu, Enrico Schlesinger and Lidia Stoppino.

Funding Open access funding provided by Università degli Studi di Pavia within the CRUI-CARE Agreement.

Open Access This article is licensed under a Creative Commons Attribution 4.0 International License, which permits use, sharing, adaptation, distribution and reproduction in any medium or format, as long as you give appropriate credit to the original author(s) and the source, provide a link to the Creative Commons licence, and indicate if changes were made. The images or other third party material in this article are included in the article's Creative Commons licence, unless indicated otherwise in a credit line to the material. If material is not included in the article's Creative Commons licence and your intended use is not permitted by statutory regulation or exceeds the permitted use, you will need to obtain permission directly from the copyright holder. To view a copy of this licence, visit http://creativecommons.org/licenses/by/4.0/.

\section{References}

1. Ådlandsvik, B.: Joins and higher secant varieties. Math. Scand. 61(2), 213-222 (1987). https://doi.org/ 10.7146/math.scand.a-12200

2. Alzati, A., Ballico, E., Ottaviani, G.: The theorem of Mather on generic projections for singular varieties. Geom. Dedicata 85(1-3), 113-117 (2001). https://doi.org/10.1023/A:1010383721612

3. Alzati, A., Ottaviani, G.: The theorem of Mather on generic projections in the setting of algebraic geometry. Manuscr. Math. 74(4), 391-412 (1992). https://doi.org/10.1007/BF02567678

4. Bastianelli, F., Ciliberto, C., Flamini, F., Supino, P.: Gonality of curves on general hypersurfaces. J. Math. Pures Appl. 9(125), 94-118 (2019). https://doi.org/10.1016/j.matpur.2019.02.016

5. Bastianelli, F., Cortini, R., De. Poi, P.: The gonality theorem of Noether for hypersurfaces. J. Algebraic Geom. 23(2), 313-339 (2014). https://doi.org/10.1090/S1056-3911-2013-00603-7

6. Ciliberto, C., Flamini, F.: On the branch curve of a general projection of a surface to a plane. Trans. Am. Math. Soc. 363(7), 3457-3471 (2011)

7. Cuzzucoli, A., Moschetti, R., Serizawa, M.: Non-uniform projections of surfaces in $P^{3}$. Le matematiche, vol. LXXII (2017)

8. De. Poi, P.: On first order congruences of lines of $P^{4}$ with a fundamental curve. Manuscr. Math. 106(1), 101-116 (2001). https://doi.org/10.1007/PL00005882

9. De. Poi, P.: Congruences of lines with one-dimensional focal locus. Port. Math. (N.S.) 61(3), 329-338 (2004)

10. Fukasawa, S.: Complete determination of the number of Galois points for a smooth plane curve. Rend. Semin. Mat. Univ. Padova 129, 93-113 (2013). https://doi.org/10.4171/RSMUP/129-7

11. Fukasawa, S.: Automorphism groups of smooth plane curves with many Galois points. Nihonkai Math. J. 25(1), 69-75 (2014)

12. Fukasawa, S., Takahashi, T.: Galois points for a normal hypersurface. Trans. Am. Math. Soc. 366(3), 1639-1658 (2014). https://doi.org/10.1090/S0002-9947-2013-05875-8

13. Guralnick, R.M., Magaard, K.: On the minimal degree of a primitive permutation group. J. Algebra 207(1), 127-145 (1998). https://doi.org/10.1006/jabr.1998.7451

14. Guralnick, R.M., Neubauer, M.G.: Monodromy groups of branched coverings: the generic case. Contemp. Math. 186, 325-352 (1995). https://doi.org/10.1090/conm/186/02190 
15. Guralnick, R.M., Shareshian, J.: Symmetric and alternating groups as monodromy groups of Riemann surfaces. I. Generic covers and covers with many branch points. Memoirs of the American Mathematical Society vol. 189(886) (2007)

16. Harris, J.: Galois groups of enumerative problems. Duke Math. J. 46(4), 685-724 (1979)

17. Harris, J.: Curves in projective space. Séminaire de Mathématiques Supérieures, vol. 85 (1982)

18. Harris, J.: Algebraic Geometry, vol. 133. Springer-Verlag, New York (1992)

19. Hartshorne, R.: Algebraic Geometry. Graduate Texts in Mathematics, vol. 52. Springer-Verlag, New York, Heidelberg (1977)

20. Lazarsfeld, R.: Positivity in algebraic geometry. I. Ergebnisse der Mathematik und ihrer Grenzgebiete. 3. Folge. vol. 48 (2004)

21. Mather, J.N.: Generic projections. Ann. Math. 2(98), 226-245 (1973)

22. Miranda, R.: Algebraic curves and Riemann surfaces. Graduate Studies in Mathematics, vol. 5. American Mathematical Society, Providence (1995)

23. Miura, K.: Field theory for function fields of plane quintic curves. Algebra Colloq. 9(3), 303-312 (2002)

24. Miura, K., Yoshihara, H.: Field theory for function fields of plane quartic curves. J. Algebra 226(1), 283-294 (2000). https://doi.org/10.1006/jabr.1999.8173

25. Pirola, G.P., Schlesinger, E.: Monodromy of projective curves. J. Algebraic Geom. 14(4), 623-642 (2005)

26. Segre, C.: Un' osservazione sui sistemi di rette degli spazi superiori. Rendiconti del Circolo Matematico di Palermo Tomo II. pp. 148-349 (1888)

27. Sernesi, E.: Deformations of algebraic schemes. Grundlehren der Mathematischen Wissenschaften, vol. 334 (2006)

28. Tevelev, E.A.: Projectively dual varieties. J. Math. Sci. (N.Y.) 117(6), 4585-4732 (2003)

29. Yoshihara, H.: Function field theory of plane curves by dual curves. J. Algebra 239(1), 340-355 (2001)

30. Yoshihara, H.: Galois points for smooth hypersurfaces. J. Algebra 264(2), 520-534 (2003). https://doi. org/10.1016/S0021-8693(03)00235-7

31. Zak, F.L.: Tangents and secants of algebraic varieties. American Mathematical Society, Providence, RI, vol. 127 (1993)

Publisher's Note Springer Nature remains neutral with regard to jurisdictional claims in published maps and institutional affiliations. 